\title{
Contrasting clinical evidence for market authorisation of cardio-vascular devices in Europe and the USA: a systematic analysis of 10 devices based on Austrian pre-reimbursement assessments
}

\author{
Claudia Wild*, Judit Erdös and Ingrid Zechmeister
}

\begin{abstract}
Background: European medical device regulation is under scrutiny and will be re-regulated with stricter rules concerning requirements for clinical evidence for high-risk medical devices. It is the aim of this study to analyse the differences between Europe and USA in dealing with risks and benefits of new cardio-vascular devices.

Methods: Since no information is available on clinical data used by the Notified Body for CE-marking, data from Austrian pre-reimbursement assessments close to European market approval were used as proxy and compared with clinical data available at time of market approval by FDA in the USA.

Results: 10 cardio-vascular interventions with 27 newly CE approved medical devices were analysed. The time lag between market authorisation in Europe and in the USA is 3 to 7 years. Only 7 CE-marked devices also hold a FDA market approval, 7 further devices are in FDA approved ongoing efficacy trials. For 4 of the CE-marked devices the FDA market application or the approval-trial was either suspended due to efficacy or safety concerns or the approval was denied. Evidence available at time of CE-marking are most often case-series or small feasibility RCTs, while large RCTs and only in rare cases prospective cohort studies are the basis of FDA approvals. Additionally, the FDA often requires post-approval studies for high-risk devices.
\end{abstract}

Conclusions: Market authorisation based on mature clinical data deriving from larger RCTs and longer follow-ups do not only change the perspective on the risk-benefit ratio, but also secures real patient benefit and safety and assures payers of investing only in truly innovative devices.

Keywords: Approval, Market authorisation, Medical devices, Evidence based medicine/EbM, Health Technology Assessment/HTA, Cardio-vascular disease, Surgery, Safety

\section{Background}

Because of several market withdrawals due to unsafe or ineffective devices, European medical device regulation is under scrutiny and will be re-regulated with stricter rules concerning requirements for clinical evidence for class III (active) and class IIb (inactive) implantable medical devices [1]. The criticism from health care providers $[2,3]$ as well as from pre-coverage health technology assessors (HTA) and payers [4-6], but also from patient groups [5] is nurtured by the fact that unsafe

\footnotetext{
* Correspondence: Claudia.wild@hta.lbg.ac.at

Ludwig Boltzmann Institute for Health Technology Assessment (LBI-HTA), Garnisongasse 7/20, 1090 Vienna, Austria
}

devices reach the European markets with pre-mature clinical data. Re-regulation details are still under debate: It has been suggested to reduce the number of European market authorisation agencies ("Notified Bodies/NB") from the current 75 to a few certified ones to approve highly specialised devices or to even go for complete centralisation, as is the case with drugs. Another suggestion is to transparently document the approval process, the evidence requirements and the provided clinical data [7].

It is well known that new medical devices, including all high-risk cardio-vascular devices, receive the European market approval (CE/ Conformité Européenne mark) several years prior to USA market authorisation [2]. Due 
to the lack of transparency where the devices are approved and what the decentralised NBs receive as application information, the actual clinical evidence can only be speculated via clinical studies published after the CE mark was issued.

Still, because of pressure from physician groups wishing to offer patients early access to innovative medicine and from manufacturers wishing early market expansion, applications for coverage of those newly CE marked devices are submitted to reimbursement institutions only several months later [8]. In a recent analysis of seven devices from all medical disciplines, it could be shown that Austria is among the first countries where applications for uptake and inclusion into the benefit catalogue are put forward [8]. Since 2008, the Ludwig Boltzmann Institute for HTA (LBI-HTA) has been commissioned by the Austrian Ministry of Health $(\mathrm{MoH})$ to assess new hospital interventions, whereby new high-risk cardio-vascular implantable devices have accounted for one-third of all primary assessments.

The aim of this study is to analyse what evidence was available for cardio-vascular devices at the time of $\mathrm{CE}$ marking using the evidence presented in the Austrian assessments as a proxy, and to compare this data with the data available at the time of FDA (Food and Drug Administration) approval. Thereby, we intend to challenge the argument that earlier provision of new devices is always of benefit to the patient.

\section{Methods}

Since no information is available on clinical data used by the NB for CE marking and because the Austrian prereimbursement assessments are rather close to $\mathrm{CE}$ marking, we assumed that the clinical evidence - provided by the manufacturer - used for CE mark could be considered a subset of the one used for the assessments produced for deciding on including the interventions in the hospital benefit catalogue. For this study we assumed the evidence is the same. Out of all pre-reimbursement assessments we selected those that dealt with cardiovascular interventions. Since the intention of the analysis was to concentrate on the level of evidence of new medical devices, we excluded those assessments that dealt with a) interventions where no new (defined by us as max. 3-5 years prior to assessment) CE marked products were available at the time of assessment, b) expansion of established indications (new interventions) with already existing products, and c) cerebro-vascular interventions.

From the included assessments (published between 2006 and 2014) we extracted information on a) intervention, b) devices, c) indication and d) best available evidence in terms of number and types of studies and number of study participants. Furthermore, we searched for publicly available online information on $\mathrm{CE}$ marking (spring 2014) and, in cases of missing information, contacted the manufacturers directly, rather than consulting the NANDO (New Approach Notified and Designated Organisations) Database.

In the USA, new high-risk devices typically undergo a premarket approval (PMA) process based on an efficacy trial [9]. Devices can receive an IDE status (investigational device exemption) that allows the device to be used in clinical approval trials [10]. Moreover, by defining so-called HDE conditions (humanitarian device exemption), it is intended that patients with a disease manifested in fewer than 4,000 individuals benefit from a device [11]. To obtain information on the time of FDA approval and on available evidence at the time of approval, we searched for PMA, IDE, and HDE documents related to the selected interventions and devices (June to July 2014). Again, data on clinical evidence for PMA decisions and trial information were extracted.

Next, we assigned the levels of clinical evidence according to the Oxford Centre for Evidence-based Medicine hierarchy: 1: SR (systematic review) or MA (meta-analysis) based on several high quality RCTs (randomized clinical trials); 2: at least $1 \mathrm{RCT}$ of high quality; 3: CTs (controlled trials) without randomisation; 4: prospective case-control and cohort studies; 5: case reports and retrospective case series [12].

Finally, we compared the market authorisation status and the level of evidence available around the time of market authorisation between Europe and the USA. Moreover, we analysed in more detail the differences between Europe and the USA in dealing with clinical evidence and with risk-benefit evaluations.

\section{Results}

Between 2008 and 2014, 15 (out of 48) pre-reimbursement assessments dealt with cardio-vascular interventions. From those, assessments on 10 cardio-vascular interventions were finally included into the analysis according to the pre-defined criteria (Table 1).

\section{Market authorisation granted}

For the 10 cardio-vascular interventions analysed, 27 newly approved CE marked medical devices were available (see details in Table 2). Of those, only 6 devices also hold a PMA status, 1 holds the HDE status, while 7 medical devices are under IDE. For 4 of the devices, the application in the USA was either suspended due to efficacy or safety concerns before/during/after the IDE trials (Cotovance $^{\mathrm{Tn}}$, Ventana ${ }^{\mathrm{Tx}}$, Symplicity ${ }^{\mathrm{Tm}}$ ), or market authorisation was denied (Watchman ${ }^{\circ}$ ) due to safety concerns. 12 CE marked cardio-vascular devices are neither PMAapproved, nor hold an IDE status (yet), meaning that they are produced solely for the European market or the 
Table 1 Cardio-vascular interventions and medical devices, years of approval in Europe and in the USA, year of reimbursement-application in Austria (2008-2014)

\begin{tabular}{|c|c|c|c|c|c|c|}
\hline Intervention & Product/manufacturer & Indication & $\begin{array}{l}\text { Year of CE mark by } \\
\text { NB/notified body }\end{array}$ & $\begin{array}{l}\text { Year of } \\
\text { reimbursement } \\
\text { application }\end{array}$ & $\begin{array}{l}\text { Year of FDA } \\
\text { approval }\end{array}$ & $\begin{array}{l}\text { Time between EU } \\
\text { and USA approval }\end{array}$ \\
\hline \multirow{2}{*}{$\begin{array}{l}\text { 1. Percutaneous pulmonary valve } \\
\text { implantation for right } \\
\text { ventricular outflow tract } \\
\text { dysfunction in patients with } \\
\text { congenital heart defects }\end{array}$} & \multirow[t]{2}{*}{ Melody $^{\mathrm{TM}} /$ Medtronic } & \multirow{2}{*}{$\begin{array}{l}\text { Patients born with a } \\
\text { dysfunctional conduit of the } \\
\text { right ventricular outflow tract } \\
\text { (RVOT) }\end{array}$} & \multirow[t]{2}{*}{2006} & \multirow[t]{2}{*}{2008} & 2010 & \multirow[t]{2}{*}{4 years } \\
\hline & & & & & Jan: HDE Melody ${ }^{\mathrm{TM}}$ & \\
\hline \multirow{5}{*}{$\begin{array}{l}\text { 2. Percutaneous aortic valve } \\
\text { replacement/TAVI }\end{array}$} & \multirow{3}{*}{$\begin{array}{l}\text { Edwards SAPIEN XTM/Edwards } \\
\text { Lifesciences }\end{array}$} & \multirow{5}{*}{$\begin{array}{l}\text { Inoperable or high-risk patients } \\
\text { with severe aortic valve stenosis }\end{array}$} & 2010 & 2008 & 2014 & 4 years \\
\hline & & & \multirow[t]{4}{*}{2011} & \multirow{4}{*}{$\begin{array}{l}\text { Updates: } 2009 \\
\text { 2010, } 2011\end{array}$} & Jan: PMA & \multirow[t]{4}{*}{3 years } \\
\hline & & & & & CoreValve $^{\oplus}$ & \\
\hline & \multirow[t]{2}{*}{ CoreValve ${ }^{\circledast} /$ Medtronic } & & & & June: PMA & \\
\hline & & & & & Edwards SAPIEN XT'M & \\
\hline \multirow{2}{*}{$\begin{array}{l}\text { 3. Cardiac contractility } \\
\text { modulation (CCM) for heart } \\
\text { failure }\end{array}$} & \multirow[t]{2}{*}{ Optimizer ${ }^{\mathrm{TM}}$ III/Impulse Dynamics } & \multirow{2}{*}{$\begin{array}{l}\text { Patients with symptomatic heart } \\
\text { failure, NYHA Stadium } \geq I I \text { and } \\
\text { normal QRC complex in ECG }\end{array}$} & \multirow[t]{2}{*}{2007} & 2008 & \multirow[t]{2}{*}{ No PMA } & \multirow[t]{2}{*}{-} \\
\hline & & & & $\begin{array}{l}\text { Updates: 2009, } \\
2010\end{array}$ & & \\
\hline \multirow{8}{*}{$\begin{array}{l}\text { 4. Percutaneous transluminal } \\
\text { angioplasty (PTA) of periphery } \\
\text { arteries with drug-eluting } \\
\text { balloon (DEB) }\end{array}$} & \multirow{2}{*}{$\begin{array}{l}\text { In.Pact }{ }^{\mathrm{TM}} \text { Amphirion + Admiral/ } \\
\text { Medtronic }\end{array}$} & \multirow{8}{*}{$\begin{array}{l}\text { Patients with peripheral artery } \\
\text { diseases, including extracranial } \\
\text { carotid and vertebral artery } \\
\text { disease, upper extremity artery } \\
\text { disease, mesenteric artery } \\
\text { disease, renal artery disease as } \\
\text { well as lower extremity artery } \\
\text { disease }\end{array}$} & In.Pact ${ }^{\mathrm{TM}}: 2009$ & \multirow[t]{8}{*}{2013} & \multirow{8}{*}{$\begin{array}{l}2014 \\
\text { July: Lutonix DCB }{ }^{\mathrm{TM}}\end{array}$} & \multirow[t]{8}{*}{5 years } \\
\hline & & & Lutonix DCB ${ }^{\mathrm{TM}}: 2011$ & & & \\
\hline & Lutonix DCB TM/Bard-Lutonix & & Cotavance $^{\mathrm{TM}:} 2011$ & & & \\
\hline & \multirow{2}{*}{$\begin{array}{l}\text { Cotavance }^{\mathrm{TM}} / \text { Bayer Schering } \\
\text { Advance } 18 \text { PTX } / \text { Cook Medical }\end{array}$} & & \multirow{3}{*}{$\begin{array}{l}\text { Advance PTX }{ }^{\oplus:} \\
\text { 2011LEGflow }{ }^{T M}: \text { 2011/2012 }\end{array}$} & & & \\
\hline & & & & & & \\
\hline & LEGflow ${ }^{\mathrm{TM}} /$ Cardionovum & & & & & \\
\hline & ELUTAX SVTM/Aachen Resonance & & Elutax SV'M: 2013 & & & \\
\hline & Freeway ${ }^{T M} /$ Eurocor & & $\begin{array}{l}\text { Freeway }^{\mathrm{TM}} \text { : year not } \\
\text { disclosed }\end{array}$ & & & \\
\hline \multirow{4}{*}{$\begin{array}{l}\text { 5. Percutaneous transluminal } \\
\text { coronary angioplasty (PTCA) } \\
\text { with drug-eluting balloon } \\
\text { (DEB) }\end{array}$} & \multirow{2}{*}{$\begin{array}{l}\text { Dior }{ }^{\oplus} \text { PCB/EurocorSeQuent }{ }^{\bullet} \\
\text { Please DCB/Braun Melsungen AG }\end{array}$} & \multirow{4}{*}{$\begin{array}{l}\text { Patients with coronary artery } \\
\text { diseases with in-stent-restenosis } \\
\text { (ISR), ostium stenosis, stenosis of } \\
\text { small coronary vessel disease } \\
\text { (SVD) and de-novo lesion of } \\
\text { coronary vessels }\end{array}$} & Dior ${ }^{\circledR}$ PCB: 2007 & 2009 & No PMA & - \\
\hline & & & SeQuent ${ }^{\oplus}$ Please DCB: 2009 & Update: 2013 & & \\
\hline & In.Pact ${ }^{\mathrm{TM}}$ Admiral/Medtronic & & In.Pact ${ }^{\mathrm{TM}}$ Admiral: 2009 & & & \\
\hline & Cotavance ${ }^{T M} /$ Bayer Schering & & Cotavance $^{\mathrm{TM}:} 2011$ & & & \\
\hline 6. Percutaneous repair of mitral & MitraClip $₫ / A b b o t t$ & Patients with moderately severe & 2008 & 2010 & 2013 & 5 years \\
\hline $\begin{array}{l}\text { regurgitation with the } \\
\text { MitraClip }\end{array}$ & & $\begin{array}{l}\text { or severe mitral regurgitation } \\
\text { (grade } 3+\text { or } 4+\text { ); both operable }\end{array}$ & & Update: 2012 & March: PMA & \\
\hline & & and inoperable patients & & & MitraClip $\otimes / A b b o t t$ & \\
\hline 7. Renal Denervation & Symplicity ${ }^{\mathrm{TM}}$ RDN/Medtronic & Patients with therapy resistant & 2008 & 2011 & No PMA & - \\
\hline & & $\begin{array}{l}\text { hypertonia after unsuccessful } \\
\text { treatment (no blood pressure }\end{array}$ & & Update: 2012 & & \\
\hline
\end{tabular}

iac contractility

modulation (CCM) for heart

Percutaneous translumina angioplasty (PTA) of periphery arteries with drug-eluting with drug-eluting balloon

Percutaneous repair of mitra regurgitation with the

Renal Denervation

decrease) with at least 3

Update: 2012 
Table 1 Cardio-vascular interventions and medical devices, years of approval in Europe and in the USA, year of reimbursement-application in Austria (2008-2014) (Continued)

\begin{tabular}{|c|c|c|c|c|c|c|}
\hline \multirow{3}{*}{$\begin{array}{l}\text { 8. Endovascular repair of aortic } \\
\text { aneurysms }\end{array}$} & \multirow{2}{*}{$\begin{array}{l}\text { Zenith }{ }^{\circledast} \text { Fenestrated AAA } \\
\text { Endovascular Graft/Cook Medical }\end{array}$} & \multirow{3}{*}{$\begin{array}{l}\text { Patients with abdominal aortic } \\
\text { aneurysm and/or iliacal aneurysm } \\
\text { having morphology suitable for } \\
\text { endovascular repair }\end{array}$} & Zenith ${ }^{\oplus}$ Fenestrated AAA & \multirow[t]{3}{*}{2013} & 2012 & \multirow[t]{3}{*}{7 years } \\
\hline & & & & & Zenith ${ }^{\circledast}$ Fenestrated & \\
\hline & $\begin{array}{l}\text { Ventana }{ }^{\mathrm{TM}} \text { Fenestrated System/ } \\
\text { Endologix }\end{array}$ & & $\begin{array}{l}\text { Ventana }{ }^{\mathrm{TM}} \text { Fenestrated } \\
\text { System: } 2013\end{array}$ & & AAA Endovascular Graft & \\
\hline \multirow{10}{*}{$\begin{array}{l}\text { 9. Percutaneous transluminal } \\
\text { angioplasty (PTA) with drug- } \\
\text { eluting stents in peripheral } \\
\text { arterial disease, upper limb } \\
\text { and thorax }\end{array}$} & Femoropopliteal:CYPHER ${ }^{\circledast}$ Select/ & \multirow{10}{*}{$\begin{array}{l}\text { Patients with symptomatic } \\
\text { peripheral artery disease (PAD) } \\
\text { on arteria femoralis superficialis/ } \\
\text { SFA, below the knee/BTK, visceral } \\
\text { arteria, liacalarteria, upper } \\
\text { extremities }\end{array}$} & CYPHER ${ }^{\circledast}$ Select: 2005 & \multirow[t]{10}{*}{2014} & 2012 & 3 years \\
\hline & 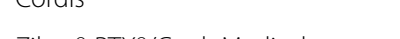 & & 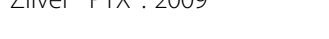 & & Zilver $^{\oplus}$ PTX & \multirow[t]{9}{*}{---} \\
\hline & Liver PIX hook Miedical & & 1" & & & \\
\hline & Innova"m/Boston Scientific & & Innova'": 2012 & & & \\
\hline & S.M.A.R.T. ${ }^{\prime} /$ Cordis & & $\begin{array}{l}\text { S.M.A.R.T.: } 2013 \\
\text { XIENCE Prime BTK: } 2011\end{array}$ & & & \\
\hline & Infrapopliteal: & & Yukon®: 2011 & & & \\
\hline & XIENCE ${ }^{\oplus}$ Prime BTK/Abbott & & PROMUS Element ${ }^{\text {TM }}$ Plus & & & \\
\hline & Yukon $\circledast /$ Translumina & & DES BTK: 2012 & & & \\
\hline & PROMUS Element ${ }^{\text {TMPlus }}$ & & & & & \\
\hline & DES BTK/Boston Scientific & & & & & \\
\hline \multirow{4}{*}{$\begin{array}{l}\text { 10. Percutaneous left atrial } \\
\text { appendage closure for the } \\
\text { prevention of } \\
\text { thromboembolic events in } \\
\text { patients with atrial fibrillation }\end{array}$} & Watchman ${ }^{\circledast}$ LAA Closure & \multirow{4}{*}{$\begin{array}{l}\text { Patients with atrial fibrillation } \\
\text { (AF)/flutter/cardiac arrhythmia/ } \\
\text { abnormal heart rhythm to } \\
\text { prevent thromboembolic events } \\
\text { such as ischaemic stroke }\end{array}$} & Watchman ${ }^{\circledast}$ LAA: 2005, & 2011 & No PMA & \multirow[t]{4}{*}{---} \\
\hline & $\begin{array}{l}\text { lechnology/Atritech-Boston } \\
\text { Scientific }\end{array}$ & & 2012 (extended use) & \multirow[t]{3}{*}{ Update: 2014} & & \\
\hline & $\begin{array}{l}\text { AMPLATZER }{ }^{T M} \text { Cardiac Plug/St. } \\
\text { Jude Medical }\end{array}$ & & $\begin{array}{l}\text { AMPLATZER }{ }^{T M} \text { Cardiac Plug: } \\
2008\end{array}$ & & & \\
\hline & $\begin{array}{l}\text { Coherex WaveCrest' }{ }^{\text {TM }} \text { LAA } \\
\text { Occlusion System/Coherex } \\
\text { Medical }\end{array}$ & & WaveCrest $^{\text {TM: }}: 2013$ & & & \\
\hline
\end{tabular}


Table 2 Evidence available at time of pre-reimbursement assessment in Austria and at time of FDA approval, levels of evidence

\begin{tabular}{|c|c|c|c|c|c|}
\hline Intervention & Product & $\begin{array}{l}\text { Highest level of evidence at } \\
\text { time of pre-reimbursement } \\
\text { assessment (Austria) }\end{array}$ & LoE & $\begin{array}{l}\text { Highest level of evidence: } \\
\text { FDA application/approval } \\
\text { (USA) }\end{array}$ & LoE \\
\hline \multirow{2}{*}{$\begin{array}{l}\text { 1. Percutaneous pulmonary valve } \\
\text { implantation for right } \\
\text { ventricular outflow tract } \\
\text { dysfunction in patients with } \\
\text { congenital heart defects }\end{array}$} & \multirow[t]{2}{*}{ Melody $^{\mathrm{TM}}$} & NB 2006 & & FDA 2010 & 4 \\
\hline & & $\begin{array}{l}2008 \text { [14]: } 4 \text { retrospective }+ \\
\text { prospective case series, } 8-68 \text { pts }\end{array}$ & 5 & $\begin{array}{l}\text { HDE approval based on } 1 \\
\text { prospective case series } 99 \text { pts, } \\
\text { requirement of } 2 \text { post-approval } \\
\text { studies with } 5 y \text { FU [13] }\end{array}$ & \\
\hline \multirow{5}{*}{$\begin{array}{l}\text { 2. Percutaneous aortic valve } \\
\text { replacement/ TAVI }\end{array}$} & CoreValve $^{\oplus}$ & 2007 & & FDA 2014 & 2 \\
\hline & \multirow[t]{4}{*}{ SAPIEN XT'M } & $\begin{array}{l}2008 \text { [16]: retrospective }+ \\
\text { prospective } 10 \text { case series, } 8-86 \\
\text { pts }\end{array}$ & 5 & \multirow{2}{*}{$\begin{array}{l}\text { CoreValve }{ }^{\oplus} \text { PMA approval based } \\
\text { on } 1 \text { RCT, } 656 \text { pts [15] (CoreValve } \\
\text { U.S. Pivotal Trial), requirement for } \\
\text { post-approval study on extreme } \\
\text { risk patients. }\end{array}$} & \\
\hline & & \multirow{2}{*}{$\begin{array}{l}2009 \text { [18]: see } 2008+4 \text { case } \\
\text { series, 12-646 pts } 2010 \text { [19]: see } \\
2008+2009+6 \text { cohort studies } \\
\text { (registries), } 4 \text { HTAs }\end{array}$} & 4 & & \\
\hline & & & 4 & \multirow{2}{*}{$\begin{array}{l}\text { SAPIEN XT'M PMA approval based } \\
\text { on } 1 \text { RCT, } 560 \text { pts [17] (PARTNER } \\
\text { II), requirement for post-approval } \\
\text { study on inoperable patients } \\
\text { cohort }\end{array}$} & 2 \\
\hline & & $\begin{array}{l}2011 \text { [20]: see } 2008+2009+ \\
2010+1 \text { RCT, } 358 \text { pts (PARTNER I) }\end{array}$ & & & \\
\hline \multirow{4}{*}{$\begin{array}{l}\text { 3. Cardiac contractility } \\
\text { modulation (CCM) for heart } \\
\text { failure }\end{array}$} & \multirow[t]{4}{*}{ Optimizer ${ }^{T M}$ III/IV } & NB 2007 & & - & - \\
\hline & & $\begin{array}{l}2008 \text { [21]: } 2 \text { RCTs, } 49 / 164 \text { pts } \\
\text { (FIX-HF-4/ OPTIMIZER) +2 case } \\
\text { series 13/25 pts) }\end{array}$ & 2 & \multirow{3}{*}{$\begin{array}{l}\text { IDE Status: Optimizer }{ }^{\mathrm{TM}} \text { III } \\
\text { OptimizerM }^{\mathrm{TM}} \text { II/IV Trial FIX-HF-5 } \\
\text { (Evaluate Safety and Efficacy of } \\
\text { the OPTIMIZER }{ }^{\oplus} \text { ) ongoing }\end{array}$} & \\
\hline & & $\begin{array}{l}2009 \text { [22]: see } 2008+1 \text { RCT- } \\
\text { Protokoll, } 428 \text { pts (FIX-HF-5/ } \\
\text { OPTIMIZER) }\end{array}$ & 2 & & \\
\hline & & 2010 [23]: see 2009 & 2 & & \\
\hline \multirow{8}{*}{$\begin{array}{l}\text { 4. Percutaneous transluminal } \\
\text { angioplasty (PTA) of periphery } \\
\text { arteries with drug-eluting } \\
\text { balloon (DEB) }\end{array}$} & In.Pact ${ }^{T M}$ Amphirion + Admiral & NB 2009/11/12/13 & & FDA 2014 & $1+3$ \\
\hline & Lutonix DCB ${ }^{\oplus}$ & \multirow{6}{*}{$\begin{array}{l}2013 \text { [25]: } 4 \text { RCTs, 50-102 pts }(2 \times \\
\text { In.Pact }{ }^{\mathrm{TM}} \text { DEBELLUM, PACIFIER, } 2 \times \\
\text { Cotovance }^{\mathrm{TM}} \text { THUNDER, FemPac) } \\
+2 \text { cohort studies (registries: } \\
\text { In.Pact }{ }^{\mathrm{TM}} \text { Admiral, In.Pact } \\
\text { Amphirion) }\end{array}$} & \multirow[t]{6}{*}{$1-2$} & \multirow{2}{*}{$\begin{array}{l}\text { Lutonix DCBं PMA based on } \\
\text { LEVANT } 2 \text { RCT, } 476 \text { pts + LEVANT } \\
2 \text { registry, } 657 \text { pts. [24] }\end{array}$} & \\
\hline & Cotavance $^{T M}$ & & & & \\
\hline & Advance 18 PTX & & & $\begin{array}{l}\text { IDE Status: In.Pact }{ }^{\mathrm{TM}} \text { Admiral } \\
\text { based on SFA I and SFA II RCT, } \\
331 \text { pts }\end{array}$ & \\
\hline & LEGflow $^{\mathrm{TM}}$ & & & \multirow{3}{*}{$\begin{array}{l}\text { No IDE Status: Cotavance }{ }^{\mathrm{TM}} \text { (IDE } \\
\text { suspended), Advance } 18 \text { PTX } \\
\text { (only EU), LEGflow } \\
\text { EU),Freeway (RAPID trial } \\
\text { (only EU) }\end{array}$} & \\
\hline & ELUTAX SVTM & & & & \\
\hline & Freeway $^{\mathrm{TM}}$ & & & & \\
\hline & Dior ${ }^{\circledast} \mathrm{PCBr}$ & \multicolumn{2}{|l|}{ NB 2007/09/11 } & - & - \\
\hline
\end{tabular}


Table 2 Evidence available at time of pre-reimbursement assessment in Austria and at time of FDA approval, levels of evidence (Continued)

5. Percutaneous transluminal ty (PTCA) with drug-eluting balloon (DEB)

SeQuent ${ }^{\oplus}$ DCB In.Pact ${ }^{\text {TM }}$ Admiral

Cotavance $^{\mathrm{TM}}$

6. Percutaneous repair of mitra regurgitation with the MitraClip

7. Renal denervation

8. Endovascular repair of aortic aneurysms

MitraClip ${ }^{\circledast / A b b o t t ~}$

Symplicity $^{\text {TM }}$ RDN/Medtronic

Zenith ${ }^{\circledast}$ Fenestrated AAA

Endovascular Graft
2009 [26]: 2 RCTs, 52/108 pts

(2x Cotavance ${ }^{\text {TM }}$ PACCOCATH I, II)

2013 [27]: 5 RCTs for ISR, 50-271 pts $\left(4 \times\right.$ SeQuent ${ }^{\circledast}$ PEPCAD II-IV + $1 \times$ Cotavance $^{\mathrm{TM}}$ PACCOCATH $\|$ FU) +1 RCT for SVD, 60 pts (Dior PICCOLETO) +1 RCT for de-novo lesions, 84 pts (SeQuent ${ }^{\oplus}$ )

NB 2008

2010 [28]: prospective case series (EVEREST I/II), 107 pts 2012 [30]:

$2010+10$ case series +1 RCT

279 pts (EVEREST II)

Ventana ${ }^{\mathrm{TM}}$ Fenestrated System

NB 2008

2011 [31]: 1 prospective case 1 RCT, 106 pts. (SYMPLICITY HTN-

2012 [33]: 2011+ 1 case series

153 pts. (FU SYMPLICITY HTN-1)

NB 2005/13

2013 [34]: 2 HTAs +4 SR based

on 1 (non-randomised)
$1-2$

based on SFA I and

SFA II RCT, 331 pts

DE Status :Dior ${ }^{\oplus}$ PCB (only

EU), SeQuent ${ }^{\oplus} \mathrm{DCB}$ : (only EU),

Cotavance $^{\mathrm{TM}}$ (IDE suspended)

FDA 2013

MitraClip ${ }^{\oplus}$ PMA approval based

on EVEREST II RCT, 279 pts,

EVEREST II high-risk registry

(EVEREST II HRR), 78 pts and

EVEREST II Cont. access registry

(REALISM HR), 853 pts [29],

requirement of 2 post-approval

studies (registries) series (SYMPLICITY HTN-1), 50 pts;

controlled study, 187 pts $+7-20$

case series, 196-368 pts
IDE Status: IDE approval study SYMPLICITY HTN-3, 535 pts. failed; 2014 approval process failed; 2014 approval process
suspended [32], IDE approval study SYMPLICITY HTN-4 ongoing

FDA 2012

Zenith ${ }^{\circledast}$ Fenestrated AAA Endovascular Graft PMA approval based on 1 (historic case-) controlled study, 42 pts [35], requirement of a long-term follow-up study.

IDE Status: Ventana ${ }^{\text {TM }}$ Fenestrated System 
Table 2 Evidence available at time of pre-reimbursement assessment in Austria and at time of FDA approval, levels of evidence (Continued)

\begin{tabular}{|c|c|c|c|c|c|}
\hline \multirow{8}{*}{$\begin{array}{l}\text { 9. Percutaneous transluminal } \\
\text { angioplasty (PTA) with drug- } \\
\text { eluting stents in peripheral } \\
\text { arterial disease, upper limb } \\
\text { and thorax }\end{array}$} & Femoropopliteal: & NB 2009/12/13 & \multirow[t]{8}{*}{$1+21+2$} & \multirow{2}{*}{$\begin{array}{l}\text { FDA } 2012 \text { Zilver }^{\oplus} \text { PTX }{ }^{\circledR} \text { PMA } \\
\text { approval based on Zilver PTX } \\
\text { randomised study, } 479 \text { pts. [37] }\end{array}$} & \multirow[t]{8}{*}{2} \\
\hline & $\begin{array}{l}\text { Zilver }^{\oplus} \text { PTX } \\
\text { Innova }\end{array}$ & 2014 [36]: Femoropopliteal & & & \\
\hline & S.M.A.R.T. ${ }^{\oplus}$ & \multirow{2}{*}{$\begin{array}{l}3 \text { RCTs, 36-479 pts }\left(1 \times \text { Zilver }^{\oplus}\right. \\
\text { PTX study, } 2 \times \text { S.M.A.R.T. } \\
\text { SCIROCCO I + II) +1 non- } \\
\text { randomised CTs, } 93 \text { pts }\left(Z_{\text {Zilver }}^{\oplus}\right) \\
+1 \text { case series, } 787 \text { pts. }\end{array}$} & & \multirow{2}{*}{$\begin{array}{l}\text { IDE Status: S.M.A.R.T. }{ }^{\oplus} \text { Vascular } \\
\text { Stent System } \\
\text { No IDE Status: Innova }{ }^{\text {TM }} \text { Peripheral } \\
\text { Vascular DES System (MAJESTIC } \\
\text { in Australia, New Zealand, EU); } \\
\text { XIENCE }{ }^{\oplus} \text { Prime BTK; }\end{array}$} & \\
\hline & Infrapopliteal: & & & & \\
\hline & XIENCE ${ }^{\oplus}$ Prime BTK & \multirow{4}{*}{ 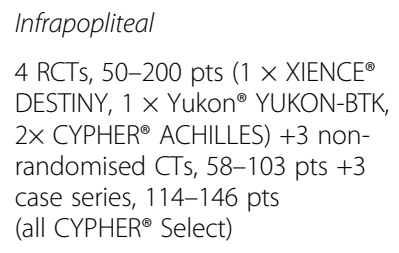 } & & \multirow{4}{*}{$\begin{array}{l}\text { Yukon }{ }^{\oplus} \text {; PROMUS } \\
\text { Element } \text { TMPlusDES BTK; CYPHER }^{\oplus} \\
\text { Select (discontinued to be } \\
\text { marketed) }\end{array}$} & \\
\hline & Yukon $^{\oplus}$ & & & & \\
\hline & PROMUS Element ${ }^{\text {TMPlusDES BTK }}$ & & & & \\
\hline & $\mathrm{CYPHER}^{\circledast}$ Select & & & & \\
\hline \multirow{4}{*}{$\begin{array}{l}\text { 10. Percutaneous left atrial } \\
\text { appendage closure for the } \\
\text { prevention of } \\
\text { thromboembolic events in } \\
\text { patients with atrial fibrillation }\end{array}$} & Watchman ${ }^{\circledast}$ LAA Closure & NB 2005/08/12/13 & $4+5$ & - & - \\
\hline & $\begin{array}{l}\text { Technology } \\
\text { AMPLATZERTM Cardiac Plug }\end{array}$ & $\begin{array}{l}2011 \text { [38]: } 4 \text { case series, 64-180 } \\
\text { pts; } 1 \text { cohort study/ registry, } 73 \\
\text { pts; } 1 \text { RCT, } 542 \text { pts (Watchman } \\
\text { PROTECT AF) }\end{array}$ & $3+2$ & $\begin{array}{l}\text { 2009: Watchman }{ }^{\oplus} \text { LAA Closure } \\
\text { Technology PMA approval based } \\
\text { on RCT, } 707 \text { pts (PROTECT AF) } \\
\text { declined [39] }\end{array}$ & \\
\hline & $\begin{array}{l}\text { Coherex WaveCrest }{ }^{\text {TM }} \text { LAA } \\
\text { Occlusion System }\end{array}$ & $\begin{array}{l}2014 \text { [40]: } 2011+3 \text { FU studies of } \\
\text { PROTECT AF + }\end{array}$ & & \multirow{2}{*}{$\begin{array}{l}\text { IDE Status: Watchman }{ }^{\circledR} \text { LAA } \\
\text { Closure, re-application with RCT } \\
\text { PREVAIL, } 407 \text { pts; AMPLATZER } \\
\text { Cardiac Plug (RESPECT), } 980 \text { pts }\end{array}$} & \\
\hline & & \multicolumn{2}{|l|}{$\begin{array}{l}3 \text { case series, } 52-86 \text { pts }+1 \\
\text { cohort study/ registry, n/a; } 2 \\
\text { prospective } C T s, 80 / 150 \text { pts }\end{array}$} & & \\
\hline
\end{tabular}


IDE will be applied for at a later stage. For 1 device (CYPHER ${ }^{\circ}$ Select), marketing was discontinued in Europe.

\section{Time lag in market authorisation}

For those 7 (6: PMA; 1: HDE) cardio-devices holding European and US licences, the time lag between market authorisation in Europe and in the USA amounted to 3 to 7 years.

\section{Levels of evidence}

The applications for coverage of new interventions and devices in cardio-vascular pathologies were submitted between 1 to 3 years after CE marking. In most of the 10 analysed interventions, the evidence available at the time of pre-reimbursement assessment was either level 4-5 (retrospective, sometimes prospective case series without control groups) or 2 (small RCTs/feasibility studies for individual devices $/ 1^{\text {st }}$ generation). Nonrandomised controlled studies (level 3) or prospectively planned registries (level 4) were seldom available. On the contrary, IDE-accepted RCTs (level 2) or - in some rare medical conditions - prospective case series or cohort studies (level 3 and 4) were the basis for FDA approval decisions in most cases (Table 2).

A detailed analysis of the efficacy and safety assessments of individual devices seems to be more informative than the levels of evidence per se. Several differences in dealing with the evidence on benefit-risk ratios could be identified and the following patterns have appeared:

\section{Requirement of extensive follow-up for high-risk} devices: Melody ${ }^{\mathrm{mm}}$ was CE marked in 2006 and has held an HDE approval since 2010. An HDE application is not required to contain the final results of scientifically valid clinical investigations, but must contain sufficient information to determine that the device does not pose an unreasonable or significant risk and that the probable benefit to health outweighs the risks [13]. Therefore, two additional post-approval studies (150 patients, follow-up of 5 years and 100 new patients with primary analysis performed at 6 months) are required by the FDA for Melody ${ }^{\mathrm{mat}}$ [41]; these studies are ongoing. No such requirements exist in Europe, since registries are voluntary. Edwards SAPIEN XT ${ }^{\mathrm{Tm}}$ and CoreValve ${ }^{\circ}$ received a CE mark in 2010 and 2011, and a PMA in 2014. The latter is based on RCTs with 560 (SAPIEN XT ${ }^{\mathrm{ma}}$ ) and 656 patients (CoreValve ${ }^{\circ}$ ) respectively. This type of clinical data was not available at the time of European market authorisation. Follow-up data from post-approval studies on inoperable and extreme risk patients is required by the FDA for both devices. No such requirements were defined at the time of EU market authorisation.
2. Early approval in Europe and later demonstration of inefficacy in RCT: Symplicity ${ }^{\mathrm{mm}}$ received CE marking in 2008 based on a safety study (SYMPLICITY HTN-1). In 2014, the FDA approval study (SYMPLICITY HTN-3) failed to meet its primary efficacy endpoint [42]. As a consequence, the manufacturer is considering a suspension of enrolment in the already ongoing SYMPLICITY HTN-4 (IDE) trial.

3. Early approvals in Europe and safety concerns in the USA: Three different devices were approved for percutaneous left atrial appendage closure for the prevention of thromboembolic events in patients with atrial fibrillation in Europe between 2005 and 2013; none of them holds a PMA status.

WATCHMAN ${ }^{\circ}$ LAA Closure Technology was denied PMA in 2009 due to safety concerns. The FDA Circulatory System Devices Panel concluded (7 in favour, 5 opposed) that although short-term efficacy had been demonstrated by the data available from the PROTECT AF trial, longer term efficacy had not been adequately demonstrated due to the lack of available long-term data [39]. In late 2013, the PREVAIL data was presented; a PMA decision is pending [43].

4. Devices of unknown value: Optimizer ${ }^{\mathrm{rw}}$ received the CE mark in 2007 based on a feasibility trial (showing no improvement in primary endpoints [21-23] and proceeded on to the IDE-approved FIX-HF-5 trial that has been running since 2011. Results are to be expected in 2015.

For PTA (percutaneous transluminal angioplasty) of peripheral arteries with drug- eluting balloon (DEB), 6 devices received the CE mark between 2009 and 2013. Only one of these (Lutonix DCB $^{\mathrm{m}}$ ) also received PMA in 2014; another (In.Pact ${ }^{\mathrm{Tx}}$ Admiral IDE) is expected to be approved by the FDA in 2015 or later. One of the DEBs, Cotavance, is $C E$ marked for the treatment of peripheral arterial disease (PAD), as well as stenotic lesions in the iliac and infrainguinal arteries, but the IDE application was suspended in 2012 due to problems concerning drug adhesion to the balloon and the according safety concerns [44].

5. Devices of critical risk-benefit-ratio: MitraClip ${ }^{\circ}$ received CE marking in 2008 on the basis of case series (EVEREST I); PMA followed in 2013. Although an RCT (EVEREST II), a prospectively planned registry (EVEREST II HRR) and a "Real World Expanded Multi-center Study" (REALISM HR) were submitted, FDA approval was cautious, with 5 votes to 3 on whether the benefits outweigh the risks and 4 votes to 5 on whether there is a reasonable assurance of efficacy. 
Two devices for the endovascular repair of aortic aneurysms received CE marking in 2005 and 2013. Only one is also approved in the USA (2012); the other (Ventana ${ }^{\mathrm{Tm}}$ ) holds an IDE, but the trial was suspended and enrolment was stopped because of a higher than expected number of re-interventions [45].

\section{Discussion}

Recent publications showed that cardiovascular devices receiving PMA (between 2000 and 2007) are often (63\%) based on non-randomised studies that lack adequate strength and may be prone to bias [46], that effectiveness endpoints are more often reported than safety endpoints, and that patient comorbidities are only incompletely reported [47]. Recalls are not uncommon, especially for those devices that have been cleared via the "substantial equivalence" process [48]. The US watchdog institution Public Citizen called medical devices in the USA "substantially unsafe" [49]. In contrast, the European debate is being led - with the exemption of the detailed analysis in [2] - on a much more general level. There is only a general demand for stricter regulation, since no data are available for analysis and there is a lack of transparency concerning which NB gave market authorisation on the basis of what type of clinical evidence.

Because of the earlier market authorisation and the lack of requirements other than the performance evaluation of medical devices in Europe (the lack of a definition of "performance" is resulting in totally different interpretations), the clinical evidence available at the time of pre-reimbursement assessments is naturally lower than some years later for market authorisation in the USA [5,50,51]. Several unsafe and ineffective devices are approved in the EU, but not in the USA [52]. The perspective of manufacturers (and of some clinical experts) that early market access provides highly innovative medicine to suffering patients [53] is held against the perspective that little is known on the effectiveness and on the risk-benefit ratio at the time of European market authorisation. Patients are put at risk and health care systems are put under pressure to invest in interventions of unknown value [6]. Since some cardiovascular devices are seemingly intended for the non-US market only, the uncertainty on their benefits and potential harms may never be resolved.

Based on our experiences from 7 years of prereimbursement assessments [54], our study contributes to the existing knowledge that not only earlier approvals are based on limited data, but that more mature data deriving from larger randomised trials and longer followups might also change the perspective on the risk-benefit ratio entirely. The two most obvious examples of devices that have been considered for market approval on both continents and where different conclusions were drawn are Symplicity ${ }^{\text {tw }}$ and Watchman ${ }^{\circ}$. Others are less visible, but still there: For Optimizer ${ }^{\mathrm{Tm}}$ (CE marked in 2007), no convincing evidence on efficacy has been demonstrated so far. In the case of Cotavance ${ }^{\mathrm{Tw}}$ (CE marked in 2011), steps towards FDA approval were suspended due to problems concerning drug adhesion to the balloon. For Ventan ${ }^{\mathrm{TI}}$ (CE marked 2013), enrolment into a trial was stopped because of a higher than expected number of re-interventions. There may even be more examples we are not aware of. Further general deficiencies are well described by Fraser et al. [2].

This study has shortcomings: The biggest limitation is the fact that no data on the clinical evidence for the $\mathrm{CE}$ marking are available; we therefore had to rely on the information on available clinical studies derived from the Austrian pre-reimbursement assessments. Nevertheless, we think it is plausible that less rather than more clinical data were available at an earlier stage, though probably not all published. Additionally, only the devices for specific indications mentioned in the pre-reimbursement applications were assessed, meaning that other cardio-devices posing problems after $\mathrm{CE}$ marking such as ProRhytm ${ }^{\circ}$ and $\mathrm{HD}$ Mesh Ablator for the treatment of Atrial Fibrillation (AF) were not included in this analysis.

\section{Conclusions}

Our conclusions are that good clinical evidence at the time of market authorisation not only secures real patient benefit and safety, but also assures payers of investing only in truly innovative devices. In addition, good clinical evidence might ease market access for manufacturers and make coverage in (hospital) benefit catalogues more predictable. There is a strong need for stricter device regulation in Europe and compulsory, long-term follow-up in order not to expose European patients to (often) premature experimental devices.

\section{Abbreviations}

AF: Atrial fibrillation; CE: Conformité Européenne; CT: Controlled trials; FDA: Food and Drug Administration; HDE: Humanitarian device exemption; IDE: Investigational device exemption; LBI-HTA: Ludwig Boltzmann Institute for HTA; MA: Meta-analysis; MoH: Ministry of health; NANDO: New approach notified and designated organisations; PAD: Peripheral arterial disease; PMA: Premarket approval; PTA: Percutaneous transluminal angioplasty; RCT: Randomized clinical trial; SR: Systematic review; USA: United States of America.

\section{Competing interests}

The authors declare that they have no competing interests.

\section{Authors' contributions}

Each author has contributed to the manuscript. CW has contributed with conception and design, analysis and interpretation of the data and writing of the paper. IZ has contributed with controlling and interpretation of the data, and commenting on draft paper. JE has contributed with collecting data and with structuring them systematically. All authors read and approved the final manuscript. 


\section{Authors' information}

CW/Claudia Wild is director of the LBI-HTA and trained in communications and social medicine; IZ/Ingrid Zechmeister is deputy director of the LBI-HTA and trained in health economics; JE/Judit Erdos is research assistant at LBI-HTA and trained in International Health Care Management.

Received: 19 September 2014 Accepted: 21 October 2014 Published: 4 November 2014

\section{References}

1. European Commission: Revision of the medical device directives. In Proposals of the European Commission. 2012 [http://ec.europa.eu/health/ medical-devices/documents/revision/index_en.htm]

2. Fraser AG, Daubert JC, Van de Werf F, Estes NAM, Smith SC, Krucoff MW, Vardas PE, Komajda M: Clinical evaluation of cardiovascular devices: principles, problems, and proposals for European regulatory reform. Report of a policy conference of the European society of cardiology. Cardiol Eur Heart J 2011, 32(13):1673-1686D.

3. Cohen D, Billingsley M: Europeans are left to their own devices. BMJ 2011, 342: 2748 .

4. Hulstaert F, Neyt M, Vinck I, Stordeur S, Huic M, Sauerland S, Kuijpers MR, Abrishami P, Vondeling H, Flamion B, Garattini S, Pavlovic M, Van Brabandt $\mathrm{H}$ : Pre-market clinical evaluations of innovative high-risk medical devices in Europe. Int J TAHC 2012, 28(3):278-284.

5. Cohen D: Patient groups accuse European parliament of putting economic interests ahead of safety on medical devices. BMJ 2013, 347:66446.

6. Storz-Pfenning P, Schmedders M, Dettloff M: Trials are needed before new devices are used in routine practice in Europe. BMJ 2013, 346:11646. Epub 18 March 2013.

7. Eikermann M, Gluud C, Perleth M, Wild C, Sauerland S, Guitarrez-Ibarluzea I, Antoine S, Demotes-Mainard J, Neugebauer EAM: Commentary: Europe needs a central, transparent, and evidence based regulation process for devices. BMJ 2013, 346:f2771. Epub 7 May 2013.

8. Krüger L, Wild C: Evidence requirements for the authorization and reimbursement of high-risk medical devices in the USA, Europe, Australia and Canada: an analysis of seven high-risk medical devices. Health Pol Technol 2014. http://dx.doi.org/10.1016/j.hlpt.2014.08.0052013.

9. U.S. Food and Drug Administration: Premarket Approval (PMA). 2014 [http://www.fda.gov/Medicaldevices/Deviceregulationandguidance/ Howtomarketyourdevice/Premarketsubmissions/Premarketapprovalpma/ Default.Htm]

10. U.S. Food and Drug Administration: Investigational Device Exemption (IDE). 2014 [http://www.fda.gov/medicaldevices/deviceregulationandquidance/ howtomarketyourdevice/investigationaldeviceexemptionide/default.htm]

11. U.S. Food and Drug Administration: Humanitarian Device Exemption (HDE) program. 2014 [http://www.fda.gov/medicaldevices/ deviceregulationandquidance/howtomarketyourdevice/ premarketsubmissions/humanitariandeviceexemption/default.htm]

12. Oxford Centre for Evidence-based Medicine: Levels of Evidence 2009. [http://www.cebm.net/oxford-centre-evidence-based-medicine-levelsevidence-march-2009/]

13. U.S. Food and Drug Administration: Medtronic Melody Transcatheter Pulmonary Valve. New Humanitarian Device Approval. 2010 [http://www.accessdata.fda.gov/cdrh_docs/pdf8/H080002b.pdf]

14. Mad P, Falkner E, Guba B, Gartlehner G: Perkutan Implantierbare Pulmonalklappen bei angeborenen Herzfehlern des rechtsventrikulären Ausflusstraktes. Vienna: Ludwig Boltzmann Institute for Health Technology Assessment; 2008. 10.

15. U.S. Food and Drug Administration: Medtronic CoreValve ${ }^{\mathrm{TM}}$ System. Summary of Safety and Effectiveness Data. 2014 [http://www.accessdata. fda.gov/cdrh_docs/pdf13/P130021b.pdf

16. Wild C, Langley T, Guba B, Gartlehner G: Minimal-Invasiver Perkutaner Aortenklappenersatz. Vienna: Ludwig Boltzmann Institute for Health Technology Assessment; 2008.

17. U.S. Food and Drug Administration: Edwards SAPIEN XT Transcatheter Heart Valve. Summary of Safety and Effectiveness. 2014 [http://www. accessdata.fda.gov/cdrh_docs/pdf13/P130009b.pdf

18. Wild C, Geiger-Gritsch S, Mittermayr T: Minimal-invasiver Perkutaner Aortenklappenersatz. Ludwig Boltzmann Institute for Health Technology Assessment. 2009. (18)/1.Update.
19. Wild C: Minimal-Invasiver Perkutaner Aortenklappenersatz. Vienna: Ludwig Boltzmann Institute for Health Technology Assessment; 2010.

20. Gottardi R, Wild C: Minimal-invasiver Perkutaner Aortenklappenersatz (mit Exkurs zu Hybrid-OPs). Vienna: Ludwig Boltzmann Institute for Health Technology Assessment; 2011.

21. Adlbrecht C, Radlberger P, Guba B, Felder-Puig R: Kardiale Kontraktilitätsmodulation bei medikamentös Therapierefraktärer Herzinsuffizienz. Vienna: Ludwig Boltzmann Institute for Health Technology Assessment; 2008.

22. Adlbrecht C, Radlberger P, Geiger-Gritsch S, Mittermayr T: Kardiale Kontraktilitätsmodulation bei medikamentös Therapierefraktärer Herzinsuffizienz. Vienna: Ludwig Boltzmann Institute for Health Technology Assessment; 2009.

23. Adlbrecht $C$, Radlberger $P$ : Kardiale Kontraktilitätsmodulation bei Patientinnen mit schwerer Herzinsuffizienz. Vienna: Ludwig Boltzmann Institute for Health Technology Assessment; 2010.

24. U.S. Food and Drug Administration: LUTONIX 035 Drug Coated Balloon PTA Catheter. Summary of Safety and Effectiveness Data. 2014 [http:// www.fda.gov/downloads/AdvisoryCommittees/CommitteesMeetingMaterials/ MedicalDevices/MedicalDevicesAdvisoryCommittee/CirculatorySystem DevicesPanel/UCM400421.pdf]

25. Warmuth M, Stumpner T: Perkutane Transluminale Angioplastie (PTA) Peripherer Arterien mit Drug-eluting Balloon (DEB). Vienna: Ludwig Boltzmann Institute for Health Technology Assessment; 2013.

26. Adlbrecht C, Radlberger P, Reiner-Theisen I, Geiger-Gritsch S, Mittermayr T: Medikamentenbeschichteter Ballonkatheter. Vienna: Ludwig Boltzmann Institute for Health Technology Assessment; 2009.

27. Hintringer K, Warmuth M: Perkutane Transluminale Koronare Angioplastie (PTCA) mit Drug-eluting Balloon (DEB). Vienna: Ludwig Boltzmann Institute for Health Technology Assessment; 2013.

28. Janatzek S, Thomas S, Mad P: Perkutane Mitralklappenintervention mittels Mitralclip Bei Mitralklappeninsuffizienz. Vienna: Ludwig Boltzmann Institute for Health Technology Assessment; 2010.

29. U.S. Food and Drug Administration: MitraClip ${ }^{\oplus}$ Clip Delivery System. Summary of Safety and Effectiveness. 2013 [http://www.accessdata.fda. gov/cdrh_docs/pdf10/P100009b.pdf]

30. Nachtnebel A, Reinsperger I: Perkutane Mitralklappenintervention Mittels Mitralclip bei Mitralklappeninsuffizienz. Vienna: Ludwig Boltzmann Institute for Health Technology Assessment; 2012.

31. Wegman $\mathrm{M}$, Thomas S, Deuber H: Perkutane Mitralklappenintervention Mittels Mitralclip bei Mitralklappeninsuffizienz. Vienna: Ludwig Boltzmann Institute for Health Technology Assessment; 2011.

32. Medtronic: Medtronic Announces U.S. Renal Denervation Pivotal Trial Fails to Meet Primary Efficacy Endpoint While Meeting Primary Safety Endpoint. 2014. Press Release.

33. Reichel M, Zechmeister-Koss I: Perkutane Renale Denervation bei Therapieresistenter Hypertonie. Vienna: Ludwig Boltzmann Institute for Health Technology Assessment; 2012.

34. Warmuth M: Endovaskuläre Versorgung Komplexer Aortenaneurysmen mit gefensterten oder verzweigten Prothesen. Vienna: Ludwig Boltzmann Institute for Health Technology Assessment: 2013.

35. U.S. Food and Drug Administration: Zenith ${ }^{\oplus}$ Fenestrated AAA Endovascular Graft. Summary of Safety and Effectiveness Data. 2012 [http://www.accessdata.fda.gov/cdrh_docs/pdf2/P020018S040b.pdf]

36. Zechmeister-Koss I, Fischer S: Medikamentenfreisetzende Stents bei peripherer arterieller Verschlusskrankheit. Vienna: Ludwig Boltzmann Institute for Health Technology Assessment; 2014.

37. U.S. Food and Drug Administration: Zilver ${ }^{\oplus}$ PTX ${ }^{\circledR}$ Drug-Eluting Peripheral Stent. Summary of Safety and Effectiveness Data. 2011 [http://www. accessdata.fda.gov/scripts/cdrh/cfdocs/cfTopic/pma/pma.cfm?num=P100022]

38. Warmuth M, Schumacher I: Perkutaner Verschluss des linken Vorhofohres zur Thrombembolieprophylaxe bei PatientInnen mit Vorhofflimmern. Vienna: Ludwig Boltzmann Institute for Health Technology Assessment; 2011.

39. U.S. Food and Drug Administration: Circulatory System Devices Panel: Meeting Summary. 2009 [http://www.fda.gov/downloads/AdvisoryCommittees/ CommitteesMeetingMaterials/MedicalDevices/MedicalDevicesAdvisoryCommittee/ CirculatorySystemDevicesPanel/UCM377356.pdf]

40. Reinsperger I, Nachtnebel A: Perkutaner Verschluss des linken Vorhofohres zur Thrombembolieprophylaxe bei PatientInnen mit Vorhofflimmern. Vienna: Ludwig Boltzmann Institute for Health Technology Assessment; 2014 
41. Armstrong AK, Balzer D, Cabalka A, Gray R, Javois A, Kreutzer J, Moore J, Rome J, Turner D, Zellers T: One Year Follow-up of the Melody ${ }^{\mathrm{T}}$ Transcatheter Pulmonary Valve Multicenter Post-Approval Study. In Presentation at American College of Cardiology/ Scientific Session. Washington, DC; 2013.

42. Medtronic: Medtronic Announces U.S. Renal Denervation Pivotal Trial Fails to Meet Primary Efficacy Endpoint While Meeting Primary Safety Endpoint. 2014 [http://newsroom.medtronic.com/phoenix.zhtml? $c=251324 \& p=$ irol-newsArticle\&ID=1889335]

43. U.S. Food and Drug Administration: Circulatory System Devices Panel: Executive Summary WATCHMAN ${ }^{\circledR}$ Left Atrial Appendage Closure Therapy. 2013 [http://www.fda.gov/AdvisoryCommittees/CommitteesMeetingMaterials/ MedicalDevices/MedicalDevicesAdvisoryCommittee/CirculatorySystem DevicesPanel/ucm152596.htm]

44. VascularNews: Is This A Setback For Drug-Eluting Balloons? 2012

45. VascularNews: Endologix Stops Enrolment in the Ventana IDE Trial. 2013.

46. Dhruva SS, Bero LA, Redberg RF: Strength of study evidence examined by the FDA in premarket approval of cardiovascular devices. JAMA 2009, 302(24):2679-2685

47. Kramer DB, Mallis E, Zuckerman BD, Zimmerman BA, Maisel WH: Premarket clinical evaluation of novel cardiovascular devices: quality analysis of premarket clinical studies submitted to the Food and Drug Administration 2000-2007. Am J Ther 2010, 17(1):2-7.

48. Zuckerman DM, Brown P, Nissen SE: Medical device recalls and the FDA approval process. Arch Intern Med 2011, 171(11):1006-1011.

49. Public Citizen: Substantially Unsafe. Medical Devices Pose Great Threat to Patients; Safeguards Must be Strengthened, Not Weakened. 2012 [http://www.citizen.org/substantially-unsafe-medical-device-report]

50. Basu S, Hassenpulg JC: Patient access to medical devices - a comparison of US and European review processes. NEJM 2012, 367(6):485-488.

51. Huot L, Decullier E, Maes-Beny K, Chapuis FR: Medical device assessment: scientific evidence examined by the French national agency for health a descriptive study. BMC Public Health 2012, 12(585).

52. U.S. Food and Drug Administration: Unsafe and Ineffective Devices Approved in the EU that were Not Approved in the US. 2012 [http://www.pharmamedtechbi.com/ /media/Supporting\%20Documents/ The\%20Gray\%20Sheet/38/20/FDA_EU_Devices_Report.pdf]

53. Boston Consulting Group: Regulation and Access to Innovative Medica Technologies: A Comparison of the FDA and EU Approval Processes and their Impact on Patients and Industry. 2012 [http://www.eucomed.org/ uploads/ModuleXtender/Newsroom/97/2012_bcg_report_regulation_ and_access_to_innovative_medical_technologies.pdf]

54. Mad P, Geiger-Gritsch S, Hinterreiter G, Mathis S, Wild C: Pre-coverage assessments of new hospital interventions in Austria: methodology and 3 years of experience. Int J TAHC 2012, 28(2):171-179.

doi:10.1186/1471-2261-14-154

Cite this article as: Wild et al:: Contrasting clinical evidence for market authorisation of cardio-vascular devices in Europe and the USA: a systematic analysis of 10 devices based on Austrian pre-reimbursement assessments.

BMC Cardiovascular Disorders 2014 14:154.

\section{Submit your next manuscript to BioMed Central and take full advantage of:}

- Convenient online submission

- Thorough peer review

- No space constraints or color figure charges

- Immediate publication on acceptance

- Inclusion in PubMed, CAS, Scopus and Google Scholar

- Research which is freely available for redistribution 